\title{
Scalable Synthesis of Bimetallic Phosphide Decorated in Carbon Nanotube Network as Multifunctional Electrocatalyst for Water Splitting
}

Dongxu Yang, Wenqiang Hou, Yingjiong Lu, Xinqiang Wang, Wanli Zhang and Yuanfu Chen*

School of Electronic Science and Engineering, and State Key Laboratory of Electronic Thin Films and Integrated Devices, University of Electronic Science and Technology of China, No. 4, Section 2, North Jianshe Road, Chengdu 610054, P. R. China

*Corresponding authors.

Tel.: +8602883202710Ｅ-mail: yfchen@uestc.edu.cn.

Number of pages: 10

Number of Figures: 12

Number of Tables: 2 


\section{Supporting Information}

(a)

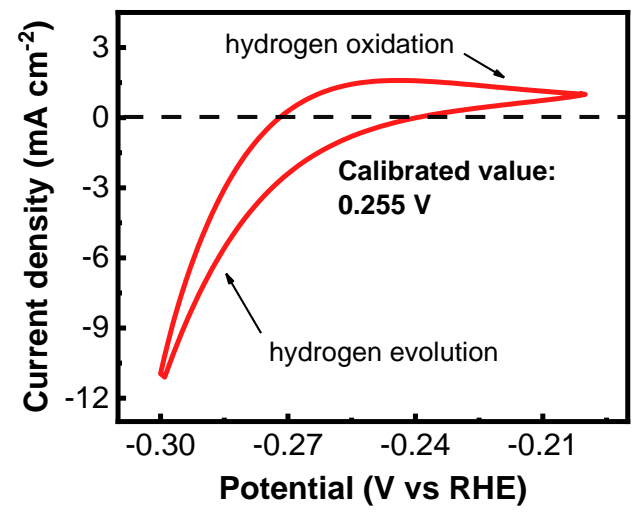

(b)

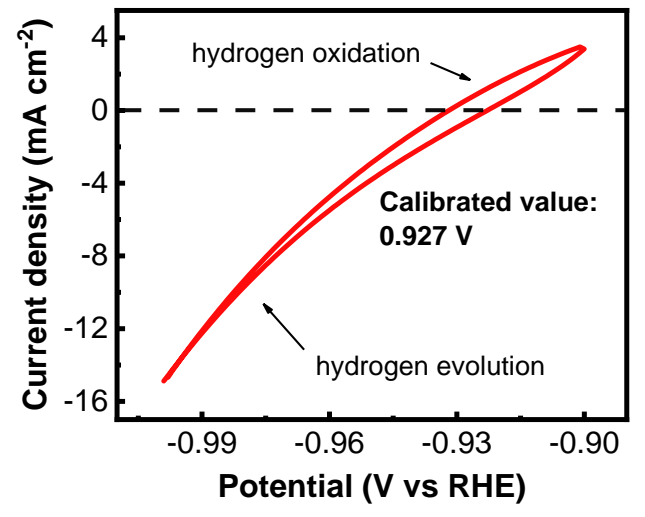

Figure S1. Voltammograms of two platinum wire electrodes as both working and counter electrodes at a scan rate of $10 \mathrm{mV} \mathrm{s}^{-1}$ in (a) $0.5 \mathrm{M} \mathrm{H}_{2} \mathrm{SO}_{4}$, (b) $1 \mathrm{M} \mathrm{KOH}$.

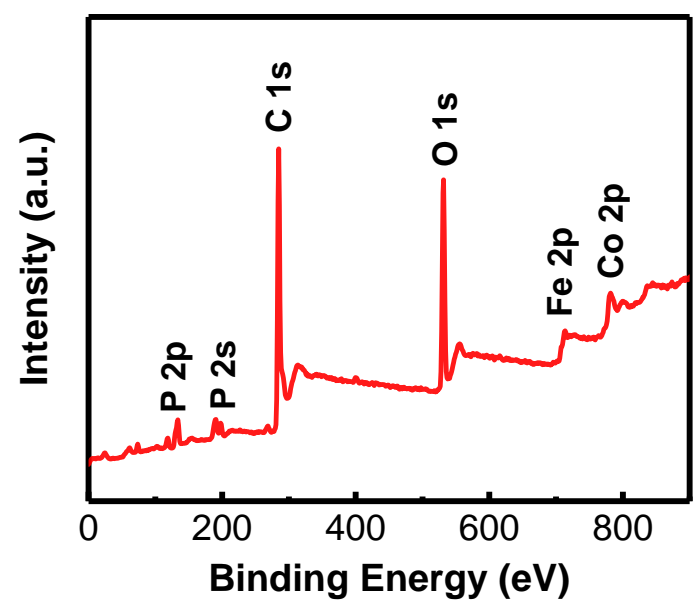

Figure S2. XPS survey spectrum of FCP-CN. 

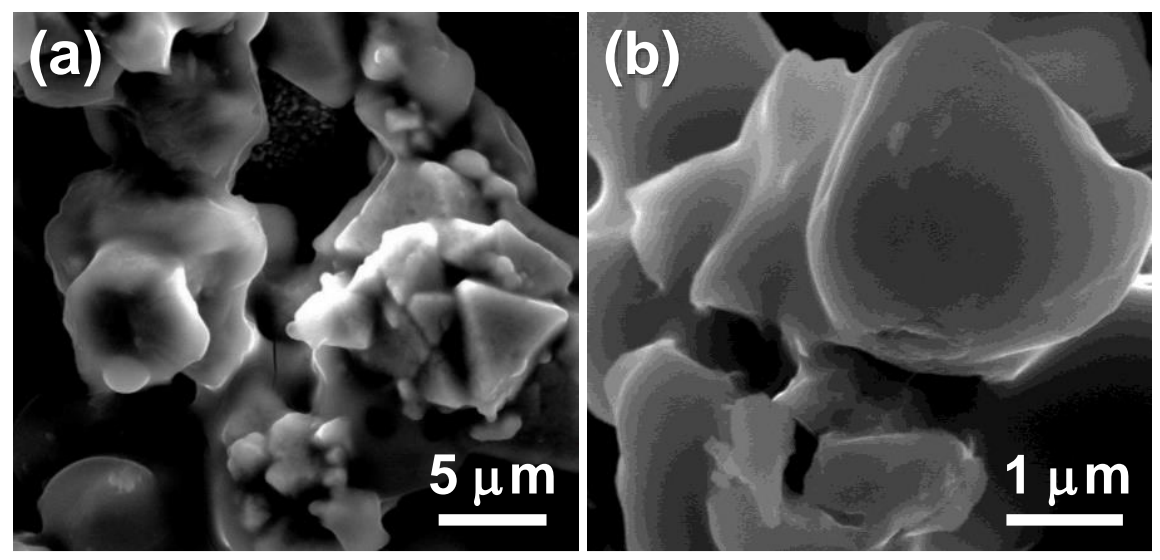

Figure S3. SEM images of bare FCP without CNT.

(a)

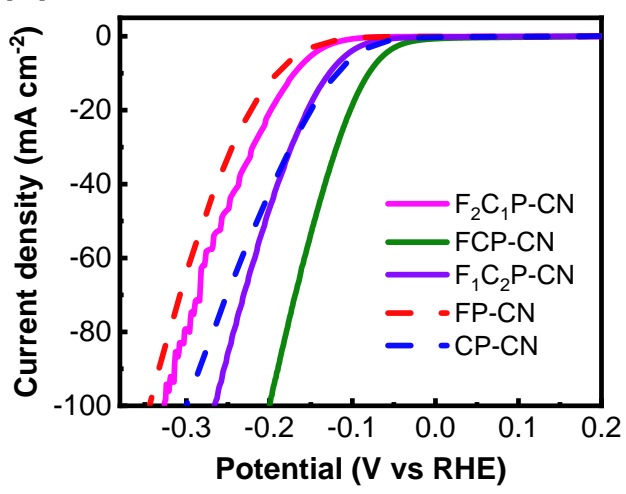

(b)

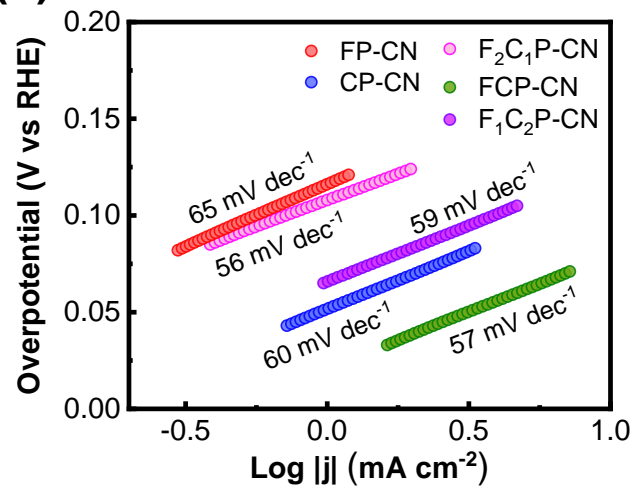

Figure S4. HER performance of $\mathrm{F}_{1} \mathrm{C}_{2} \mathrm{P}-\mathrm{CN}$, FCP-CN and $\mathrm{F}_{2} \mathrm{C}_{1} \mathrm{P}-\mathrm{CN}$, in comparison with FP-CN and CP-CN catalysts in $0.5 \mathrm{M} \mathrm{H}_{2} \mathrm{SO}_{4}$, (a) Polarization curves and (b) corresponding Tafel plots

\begin{tabular}{|c|c|c|c|c|c|}
\hline Catalyst & Electrode & $\begin{array}{l}\text { Tafel Slope } \\
\left(\mathrm{mV} \mathrm{dec}{ }^{-1}\right)\end{array}$ & $\begin{array}{c}\eta_{10} \\
(\mathrm{mV})\end{array}$ & Electrolyte & Ref \\
\hline \multirow{2}{*}{ FCP-CN } & \multirow{2}{*}{ GCE } & 57 & 80 & $0.5 \mathrm{M} \mathrm{H}_{2} \mathrm{SO}_{4}$ & \multirow{2}{*}{$\begin{array}{l}\text { This } \\
\text { work }\end{array}$} \\
\hline & & 71 & 178 & $1.0 \mathrm{M} \mathrm{KOH}$ & \\
\hline eNi-P/NCN & GCE & 69 & 190 & $1.0 \mathrm{M} \mathrm{KOH}$ & [1] \\
\hline
\end{tabular}




\begin{tabular}{|c|c|c|c|c|c|}
\hline \multirow{2}{*}{$\mathrm{CoTe}_{2} @ \mathrm{NCNTF}$} & \multirow{2}{*}{ GCE } & 62 & 240 & $0.5 \mathrm{M} \mathrm{H}_{2} \mathrm{SO}_{4}$ & \multirow{2}{*}[2]{} \\
\hline & & 83 & 208 & $1.0 \mathrm{M} \mathrm{KOH}$ & \\
\hline $\mathrm{Ni}_{2} \mathrm{P} / \mathrm{CoP} \mathrm{NP}$ & GCE & 64 & 105 & $0.5 \mathrm{M} \mathrm{H}_{2} \mathrm{SO}_{4}$ & [3] \\
\hline \multirow{2}{*}{$\mathrm{NiSe}_{2} @ \mathrm{NG}$} & \multirow{2}{*}{ GCE } & 36 & 201 & $0.5 \mathrm{M} \mathrm{H}_{2} \mathrm{SO}_{4}$ & \multirow{2}{*}{ [4] } \\
\hline & & 74 & 248 & $1.0 \mathrm{M} \mathrm{KOH}$ & \\
\hline \multirow{2}{*}{$\mathrm{MoSe}_{2}-\mathrm{NiSe}$} & \multirow{2}{*}{ GCE } & 76 & 154 & $0.5 \mathrm{M} \mathrm{H}_{2} \mathrm{SO}_{4}$ & \multirow{2}{*}[5]{} \\
\hline & & 81 & 180 & $1.0 \mathrm{M} \mathrm{KOH}$ & \\
\hline \multirow[b]{2}{*}{$\mathrm{Co}_{0.6} \mathrm{Fe}_{0.4} \mathrm{P}$} & \multirow[b]{2}{*}{ GCE } & 60 & 97 & $0.5 \mathrm{M} \mathrm{H}_{2} \mathrm{SO}_{4}$ & \multirow[b]{2}{*}[6]{} \\
\hline & & 61 & 133 & $1.0 \mathrm{M} \mathrm{KOH}$ & \\
\hline FeCo@FeCoP@C & Carbon paper & 60 & 65 & $0.5 \mathrm{M} \mathrm{H}_{2} \mathrm{SO}_{4}$ & {$[7]$} \\
\hline CF@FeCoP/NC & Carbon fabric & 75 & 89 & $1.0 \mathrm{M} \mathrm{KOH}$ & [8] \\
\hline \multirow{2}{*}{$\mathrm{NiCoP} / \mathrm{rGO}$} & \multirow{2}{*}{ Carbon paper } & 45 & 42 & $0.5 \mathrm{M} \mathrm{H}_{2} \mathrm{SO}_{4}$ & \multirow{2}{*}{ [9] } \\
\hline & & 124 & 209 & $1.0 \mathrm{M} \mathrm{KOH}$ & \\
\hline $\mathrm{FeCoP} / \mathrm{C} \mathrm{NS}$ & $\mathrm{Ni}$ foam & 107 & 55 & $1.0 \mathrm{M} \mathrm{KOH}$ & [10] \\
\hline Ni-Co-P HNB & $\mathrm{Ni}$ foam & 46 & 107 & $1.0 \mathrm{M} \mathrm{KOH}$ & {$[11]$} \\
\hline
\end{tabular}

Table S1 Comparison of HER performance of recently reported transition-metalbased catalysts
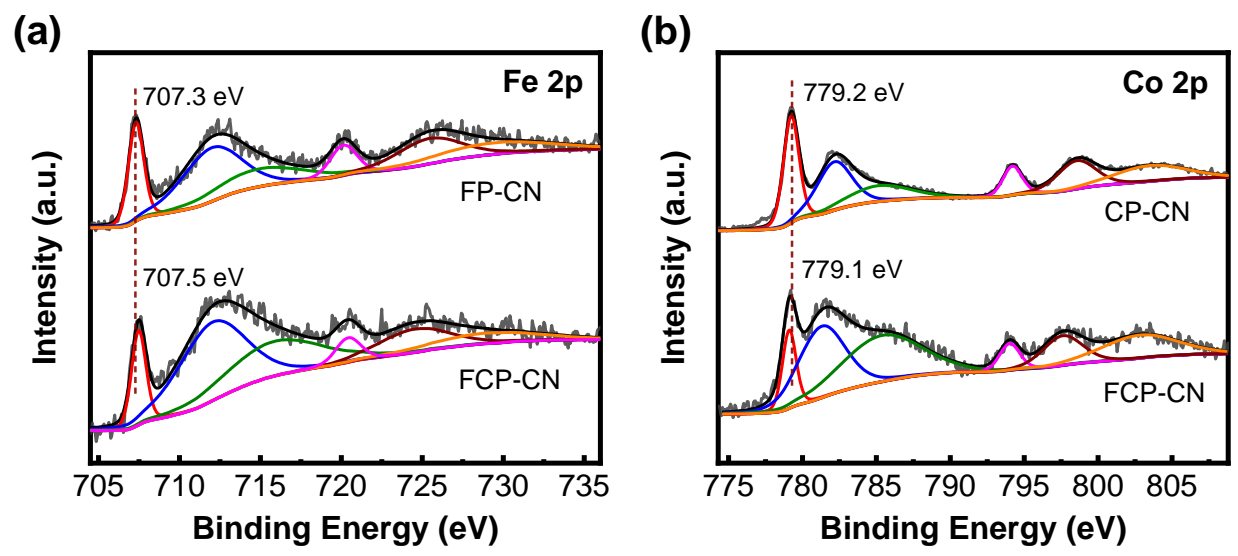
Figure S5. High-resolution XPS spectra of (a) Fe 2p for FP-CN and FCP-CN, and (b) Co $2 \mathrm{p}$ for CP-CN and FCP-CN

\begin{tabular}{|c|c|c|c|c|}
\hline Catalyst & Electrode & $\begin{array}{l}\text { Tafel Slope } \\
\left(\mathrm{mV} \operatorname{dec}^{-1}\right)\end{array}$ & $\begin{array}{c}\eta_{10} \\
(\mathrm{mV})\end{array}$ & Ref \\
\hline FCP-CN & GCE & 38 & 323 & $\begin{array}{r}\text { This } \\
\text { work }\end{array}$ \\
\hline CoP@NPCSs & GCE & 103 & 350 & {$[12]$} \\
\hline $\mathrm{Ni}_{2} \mathrm{P} / \mathrm{CoP} \mathrm{NP}$ & GCE & 69 & 320 & {$[3]$} \\
\hline $\mathrm{CoP} / \mathrm{NCNHP}$ & GCE & 70 & 310 & {$[13]$} \\
\hline $\mathrm{CoTe}_{2} @ \mathrm{NCNTFs}$ & GCE & 58 & 330 & [2] \\
\hline $\mathrm{FeNi}-\mathrm{P} / \mathrm{NCN}$ & GCE & 72 & 240 & {$[1]$} \\
\hline FeCoNi@FeNC & GCE & 57 & 330 & {$[14]$} \\
\hline $\mathrm{Co}_{0.6} \mathrm{Fe}_{0.4} \mathrm{P}$ & GCE & 48 & 298 & {$[6]$} \\
\hline $\mathrm{NiCoP} / \mathrm{rGO}$ & Carbon paper & 66 & 270 & [9] \\
\hline CF@FeCoP/NC & Carbon fabric & 57 & 228 & [8] \\
\hline $\mathrm{FeCo} / \mathrm{C} \mathrm{NS}$ & $\mathrm{Ni}$ foam & 74 & 219 & {$[10]$} \\
\hline $\mathrm{Ni}-\mathrm{Co}-\mathrm{P}$ HNBs & $\mathrm{Ni}$ foam & 76 & 270 & {$[11]$} \\
\hline
\end{tabular}

Table S2 Comparison of OER performance of recently reported transition-metalbased catalysts in alkaline medium 


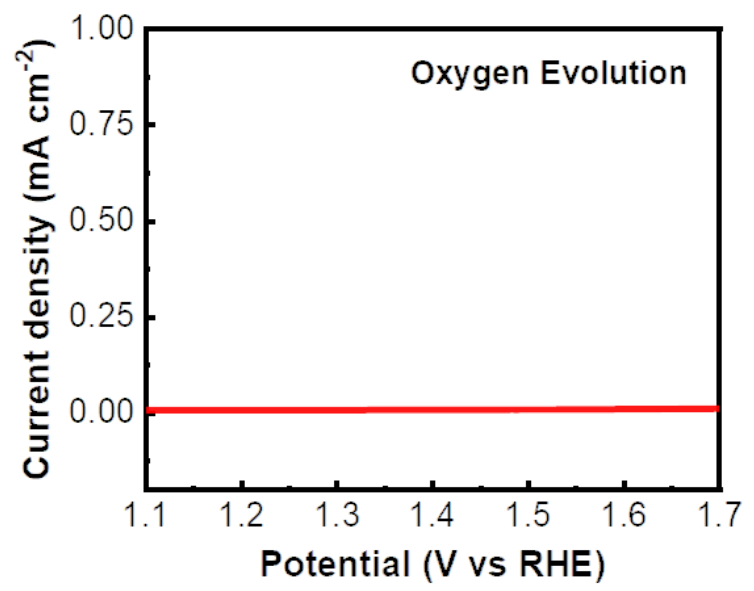

Figure S6. OER performance of FCP-CN in $0.5 \mathrm{M} \mathrm{H}_{2} \mathrm{SO}_{4}$
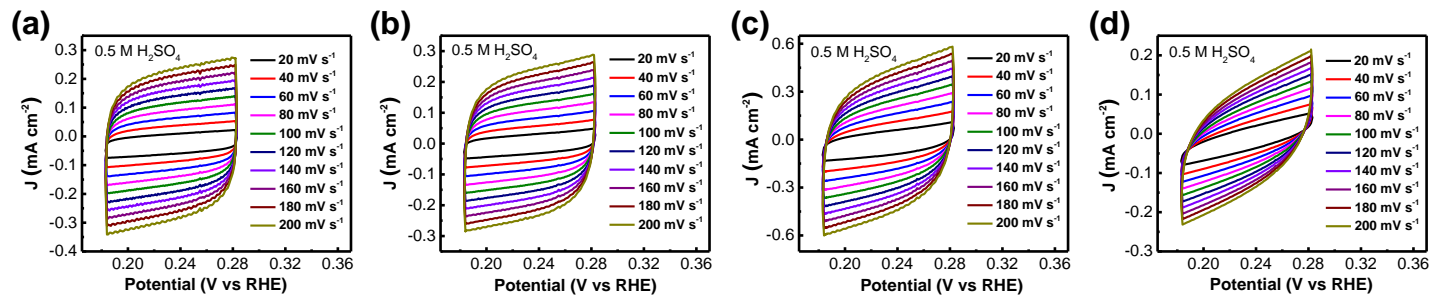

Figure S7. Cyclic voltammogram curves of (a) FP-CN, (b) CP-CN, (c) FCP-CN and (d) FCP with various scan rate $\left(20-200 \mathrm{mV} \mathrm{s}^{-1}\right)$ in $0.5 \mathrm{M} \mathrm{H}_{2} \mathrm{SO}_{4}$.
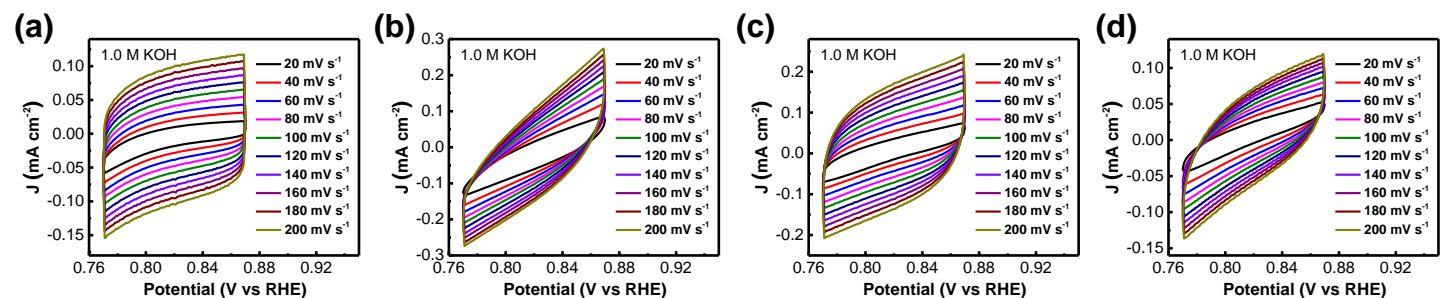

Figure S8. Cyclic voltammogram curves of (a) FP-CN, (b) CP-CN, (c) FCP-CN and (d) FCP with various scan rate $\left(20-200 \mathrm{mV} \mathrm{s}^{-1}\right)$ in $1.0 \mathrm{M} \mathrm{KOH}$. 
(a)

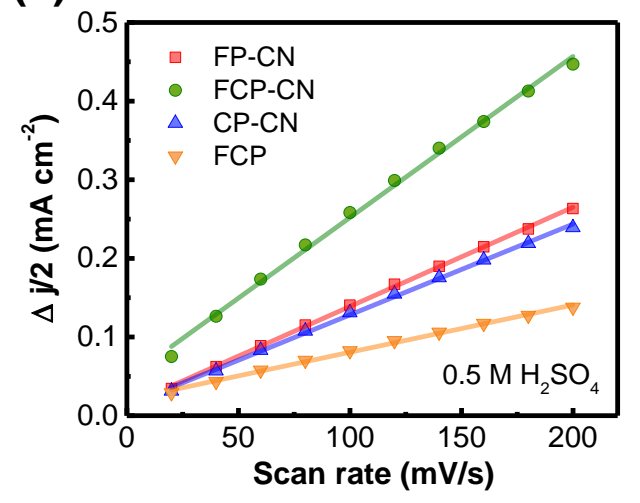

(b)

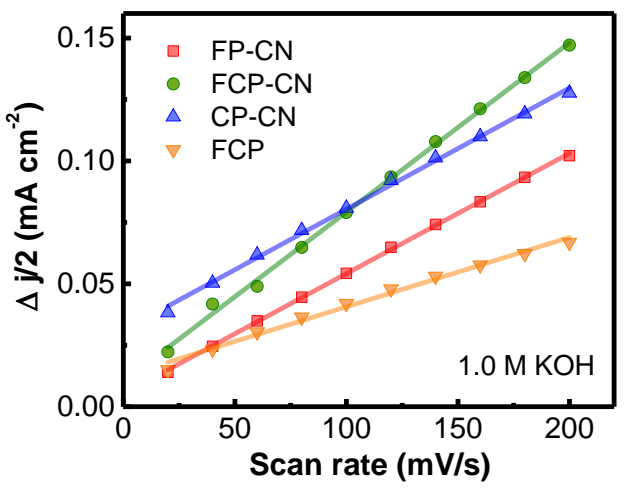

Figure S9. Linear correlation of current density variation against CV scan rate in (a) $0.5 \mathrm{M} \mathrm{H}_{2} \mathrm{SO}_{4}$ and (b) $1.0 \mathrm{M} \mathrm{KOH}$ within non-Faradaic region, reflecting the $C_{d l}$ and ECSA.

(a)

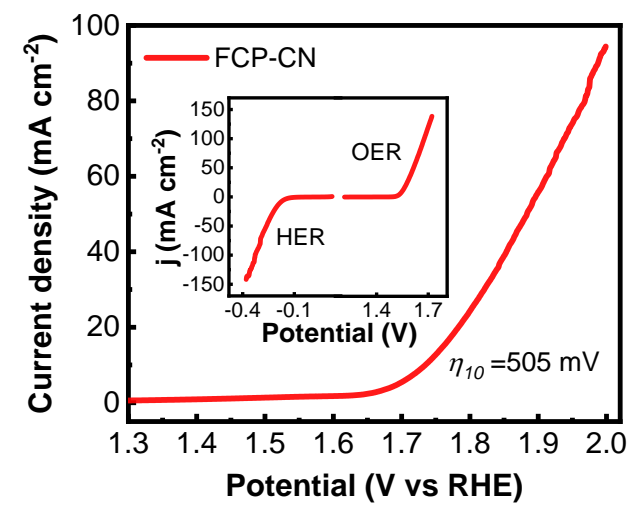

(b)

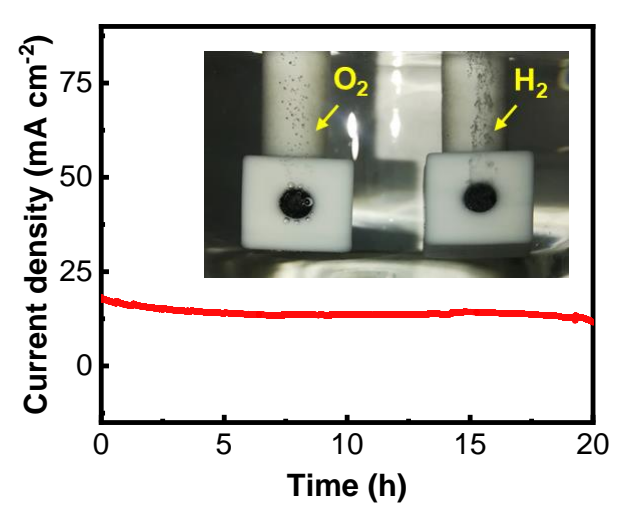

Figure S10. Overall water splitting in 1.0 M KOH: (a) polarization curve for overall water splitting; inset: polarization curves for HER and OER; (b) amperometric i-t curve at the overpotential of $550 \mathrm{mV}$; inset: digital photos for the two-electrode setup. 


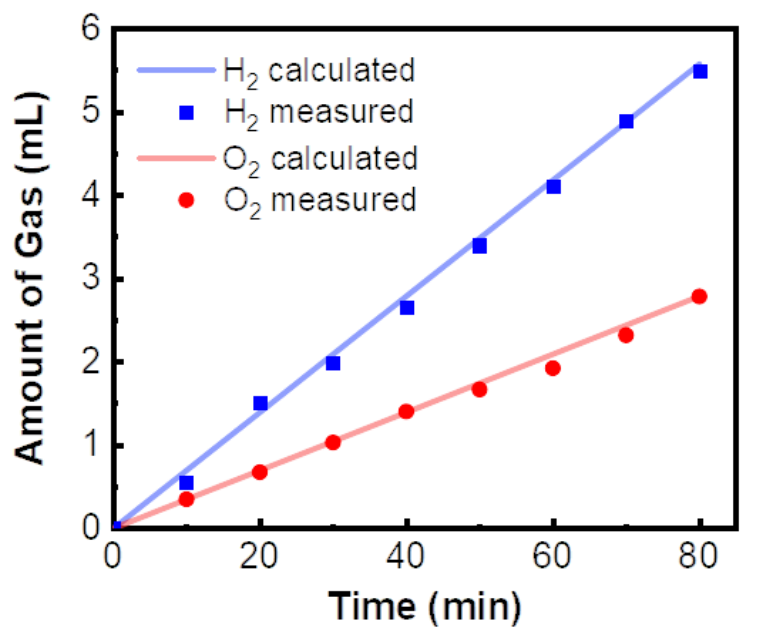

Figure S11. The experimentally measured amount of $\mathrm{H}_{2}$ and $\mathrm{O}_{2}$ in comparison with the theoretically calculated values at $10 \mathrm{~mA} \mathrm{~cm}^{-2}$.

Calculation of Faraday Efficiency: The amount of $\mathrm{H}_{2}$ and $\mathrm{O}_{2}$ can be calculated from the measured volumes applying the ideal gas law. And the theoretical quantity of $\mathrm{H}_{2}$ and $\mathrm{O}_{2}$ can be easily calculated through Faraday law based on the fix current. Therefore, the Faradic efficiency can be calculated by using the following equation:

$$
F E=\frac{V / V m}{Q /(n * F)}
$$

where $V$ is the volume of gas experimentally evolved by the reaction, $V_{m}$ is the molar volume (24.5 $\left.\mathrm{L} \mathrm{mol}^{-1}, \mathrm{RT}\right), Q$ is the total quantity of electric charge $(\mathrm{C}), F$ is the Faradaic constant $\left(96485.3 \mathrm{C} \mathrm{mol}^{-1}\right)$, and $n$ is the mole number of electrons transferred during the reaction while it is generating one mole of gas. 

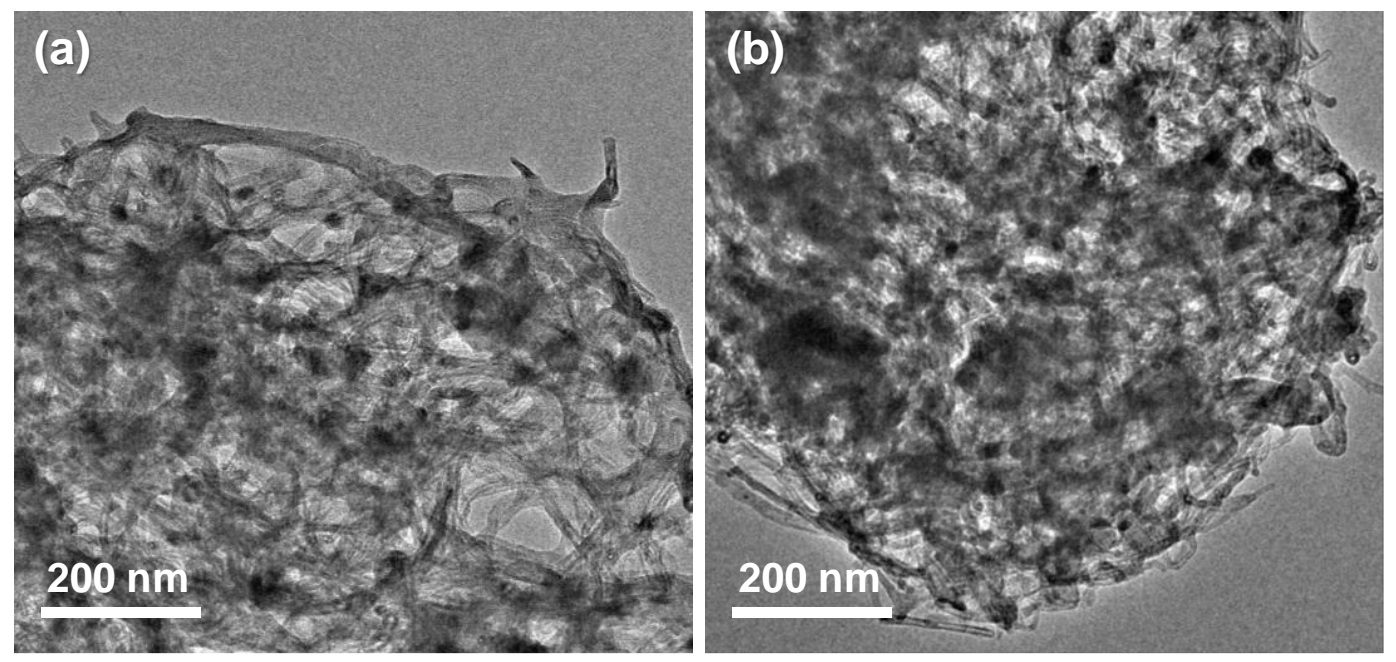

Figure S12. TEM images of FCP-CN after $1000 \mathrm{CV}$ cycles of (a) HER and (b) OER process

\section{References:}

1. Wu, Y, Tao, X, Qing, Y, Xu, H, Yang, F, Luo, S, Tian, C, Liu, M, Lu, X, Cr-Doped FeNi-P Nanoparticles Encapsulated Into N-Doped Carbon Nanotube as a Robust Bifunctional Catalyst for Efficient Overall Water Splitting. Adv Mater 2019, 31 (15), 1900178, DOI 10.1002/adma.201900178.

2. Wang, X, Huang, X, Gao, W, Tang, Y, Jiang, P, Lan, K, Yang, R, Wang, B, Li, R, Metal-Organic Framework Derived $\mathrm{CoTe}_{2}$ Encapsulated in Nitrogen-Doped Carbon Nanotube Frameworks: A HighEfficiency Bifunctional Electrocatalyst for Overall Water Splitting. J Mater Chem A 2018, 6 (8), 3684-3691, DOI 10.1039/C7TA10728E.

3. Liang, X, Zheng, B, Chen, L, Zhang, J, Zhuang, Z, Chen, B, Mof-Derived Formation of $\mathrm{Ni}_{2} \mathrm{P}-\mathrm{CoP}$ Bimetallic Phosphides with Strong Interfacial Effect Toward Electrocatalytic Water Splitting. ACS Appl Mater Inter 2017, 9 (27), 23222-23229, DOI 10.1021/acsami.7b06152.

4. Li, W, Yu, B, Hu, Y, Wang, X, Yang, D, Chen, Y, Core-Shell Structure of $\mathrm{NiSe}_{2}$ Nanoparticles@Nitrogen-Doped Graphene for Hydrogen Evolution Reaction in both Acidic and Alkaline Media. ACS Sustain Chem Eng 2019, 7 (4), 4351-4359, DOI 10.1021/acssuschemeng.8b06195.

5. Liu, C, Wang, K, Zheng, X, Liu, X, Liang, Q, Chen, Z, Rational Design of MoSe $2-\mathrm{NiSe}_{\text {Carbon }}$ Heteronanostructures for Efficient Electrocatalytic Hydrogen Evolution in Both Acidic and Alkaline Media. Carbon 2018, 139, 1-9, DOI 10.1016/j.carbon.2018.06.034.

6. Lian, Y, Sun, H, Wang, X, Qi, P, Mu, Q, Chen, Y, Ye, J, Zhao, X, Deng, Z, Peng, Y, Carved Nanoframes of Cobalt-Iron Bimetal Phosphide as a Bifunctional Electrocatalyst for Efficient Overall Water Splitting. Chem Sci 2019, 10 (2), 464-474, DOI 10.1039/c8sc03877e.

7. Zhang, J, Li, M, Liang, X, Zhuang, Z, Multishelled FeCo@FeCoP@C Hollow Spheres as Highly Efficient Hydrogen Evolution Catalysts. ACS Appl Mater Inter 2018, 11 (1), 1267-1273, DOI 10.1021/acsami.8b17612.

8. Lin, R, Lei, H, Ruan, D, Jiang, K, Yu, X, Wang, Z, Mai, W, Yan, H, Solar-Powered Overall Water 
Splitting System Combing Metal-Organic Frameworks Derived Bimetallic Nanohybrids Based Electrocatalysts and One Organic Solar Cell. Nano Energy 2019, 56, 82-91, DOI 10.1016/j.nanoen.2018.10.058.

9. Li, J, Yan, M, Zhou, X, Huang, Z, Xia, Z, Chang, C, Ma, Y, Qu, Y, Mechanistic Insights On Ternary $\mathrm{Ni}_{2-x} \mathrm{Co}_{x} \mathrm{P}$ for Hydrogen Evolution and their Hybrids with Graphene as Highly Efficient and Robust Catalysts for Overall Water Splitting. Adv Funct Mater 2016, 26 (37), 6785-6796, DOI 10.1002/adfm.201601420.

10. Xiang, R, Duan, Y, Tong, C, Peng, L, Wang, J, Shah, SSA, Najam, T, Huang, X, Wei, Z, SelfStanding $\mathrm{FeCo}$ Prussian Blue Analogue Derived $\mathrm{FeCo} / \mathrm{C}$ and $\mathrm{FeCoP} / \mathrm{C}$ Nanosheet Arrays for CostEffective Electrocatalytic Water Splitting. Electrochim Acta 2019, 302, 45-55, DOI 10.1016/j.electacta.2019.01.170.

11. Hu, E, Feng, Y, Nai, J, Zhao, D, Hu, Y, Lou, XWD, Construction of Hierarchical Ni-Co-P Hollow Nanobricks with Oriented Nanosheets for Efficient Overall Water Splitting. Energ Environ Sci 2018, 11 (4), 872-880, DOI 10.1039/C8EE00076J.

12. Wu, K, Chen, Z, Cheong, W, Liu, S, Zhu, W, Cao, X, Sun, K, Lin, Y, Zheng, L, Yan, W, Pan, Y, Wang, D, Peng, Q, Chen, C, Li, Y, Toward Bifunctional Overall Water Splitting Electrocatalyst: General Preparation of Transition Metal Phosphide Nanoparticles Decorated N-Doped Porous Carbon Spheres. ACS Appl Mater Inter 2018, 51 (10), 44201-44208, DOI 10.1021/acsami.8b14889.

13. Pan, Y, Sun, K, Liu, S, Cao, X, Wu, K, Cheong, W, Chen, Z, Wang, Y, Li, Y, Liu, Y, Wang, D, Peng, Q, Chen, C, Li, Y, Core-Shell ZIF-8@ZIF-67-Derived CoP Nanoparticle-Embedded N-Doped Carbon Nanotube Hollow Polyhedron for Efficient Overall Water Splitting. J Am Chem Soc 2018, 140 (7), 2610-2618, DOI 10.1021/jacs.7b12420.

14. Zhang, Q, Webster, RF, Cheong, S, Tilley, RD, Lu, X, Amal, R, Ultrathin Fe-N-C Nanosheets Coordinated Fe-Doped CoNi Alloy Nanoparticles for Electrochemical Water Splitting. Part Part Syst Char 2018, 36, 252-261, DOI 10.1002/ppsc.201800252. 